\title{
Using "Unit-Function-Object" Approach to Solve Main Problems in Ontology Engineering
}

\author{
Anna A. Kondratenko, Sergei I. Matorin \\ Institute of Engineering Technology and Natural Science \\ Belgorod State University \\ Belgorod, Russia \\ a.kondratenko-sl@yandex.ru
}

\begin{abstract}
This article is devoted to the main problems in the one of the most actual research area in knowledge management ontology engineering. An original approach to solving the problems of applied ontologies building, inference on them, ontology mapping and merging, which is based on the "UnitFunction-Object" approach, is proposed. The main components of the corresponding methods with the basis of UFO approach and UFO domain modeling principles are considered.
\end{abstract}

Keywords-ontology; ontology engineering; UFO approach; UFO model; ontologies mapping; ontologies merging; RDF

\section{INTRODUCTION}

Nowadays, in the context of increasing role of organizational knowledge in the enterprises competition, various methods and approaches in knowledge management are actively developing. The one of the most actual and indemand among them is such a scientific direction as ontology engineering. This term means the theory and technologies for the building of ontologies of various levels, including ontologies for solving specific applied problems of knowledge management in a given domain.

Ontology in the classical mean (according to Gruber) is an «explicit specification of conceptualization» [1]. In other words, ontology is a detailed specification of the structure of a particular problem area. There are many different definitions of the term «ontology» in modern publications, however, scientists and experts are agreed, that such structures are effectively applied for solving some complex problems in knowledge management. For example, according to [2], the possible options for using ontologies are: communication (development and sharing of the language, which is formally accepted by domain experts); integration of distributed and dissimilar information resources; data analysis and logical inference in terminology.

Expanding the range of tasks solved with the help of ontologies leads to the need of development effective methods for solving the main problems of ontological engineering. One of the approaches to solving most of these problems is the integration of ontology engineering tools and the "UnitFunction-Object" approach.

\section{MAIN TASKS AND PROBLEMS OF ONTOLOGY ENGINEERING}

\section{A. Applied ontology building}

One of the main tasks of ontology engineering is the organization of an effective process of building new applied ontologies. Scientists note that ontologies development is often a complex, lengthy and costly process that requires significant efforts from participants [3].

In particular, as a rule, there are certain requirements to participants of ontology building process. To achieve maximum results, the team must include developers with professional experience in the knowledge management technologies area: from methods of extracting knowledge to structuring and formalization [4]. Also it is recommended to involve in the process of ontology building domain experts, who in general are not professionals in the knowledge management. This aspect increases the number of participants.

In the process of ontology development practically independently of selected technique the part of "manual" work of domain experts and developers are very large. Most operations to identify concepts and their relationships, build taxonomies and refine the prototype of the ontology are done without the help of any automated means. This makes ontology development very difficult and increases the probability that the resulting ontology will not be adequate to the original requirements

Because of the increasing role of ontologies in the semantic web and intelligent information systems progress, the question of formalizing and further automating the process of domain ontologies building becomes topical. Nowadays there are no effective methods and tools that allow you to obtain prototypes of domain ontologies and use them in information systems with minimal effort.

This means that the actual task is to improve the existing and develop new, more effective methods for constructing applied ontologies.

\section{B. Mapping ontologies}

Another important task is ontologies mapping - the process of establishing a correspondence between ontologies or identifying semantic links of similar elements from different 
ontologies [5]. In other words, mapping ontology A with ontology B means that for each concept in first ontology a corresponding concept with the same or similar semantics in second ontology, and on the contrary.

Finding the correspondences and differences between compared ontologies is the key to solving such problems in the ontology matching area, like alignment, merging, modification to ensure homogeneity of several ontologies. Aspects of ontologies mapping and matching are extremely actual to solving practical problems, when it is necessary to operate with several applied ontologies for the same or related subject areas.

\section{INTEGRATION UFO APPROACH MEANS WITH ONTOLOGY ENGINEERING}

\section{A. Fundamental aspects of 'Unit-Function-Object' approach}

To solve the previously listed problems the means of the "Node-Function-Object" approach (UFO approach) is proposed to use. The main principles of the UFO approach and based on it UFO analysis and UFO modeling, have been developed by russian scientists and have been successfully tested for a variety of applications in the areas of system analysis, domain modeling and knowledge management [6-9].

The base of the UFO-approach and UFO-analysis is representation of one or another system in the form of a threeelement construction "Unit-Function-Object" (UFO). In this context system as a Unit is a structural element, a "crossroads" of all its connections. The Function represents the system as a functional element that plays a given role in maintaining the supersystem by balancing this Node. An Object represents a system in the form of a specific formation, a substantial element that realizes a Function. Combinations of similar UFO elements called system-object models (UFO models) [10]. Thus, using of system-object UFO analysis provides a combination of functional and object modeling in one model.

The representation of some domain in the form of the UFO model provides the most holistic, systemic representation of its purpose, entity, position in the structure of the supersystem, and links to other elements in general, because the UFO model contains information about both the structural and functional characteristics of the modeled system.

UFO model as a result of applying UFO analysis is an adequate and fairly complete domain model. It is easy to identify in a such model the main concepts (terms, entities), their characteristics (attributes, properties), its role in the system and the interrelationships of such concepts. The UFO model reflects only the terms that are necessary for describing selected domain, and precisely in the sense that they are used directly in the this domain. The development of the domain UFO model does not require significant labor and time costs.

The UFO analysis method allows to include in the resulting model information about the domain, presented from different points of view, which provides the maximum possible objectivity of reflection of knowledge about domain. The resulting UFO model has the scalability property and can be easily modified when domain is changed. Unlike other common methodologies of system and object modeling, the
UFO approach has wide possibilities for formalization. These features of the UFO approach open up new opportunities for solving ontology engineering problems.

\section{B. Ontology building with UFO approach means}

In particular, an original method of applied ontologies development based on UFO domain models was proposed recently [11]. The main components of this method are shown in the diagram (figure).

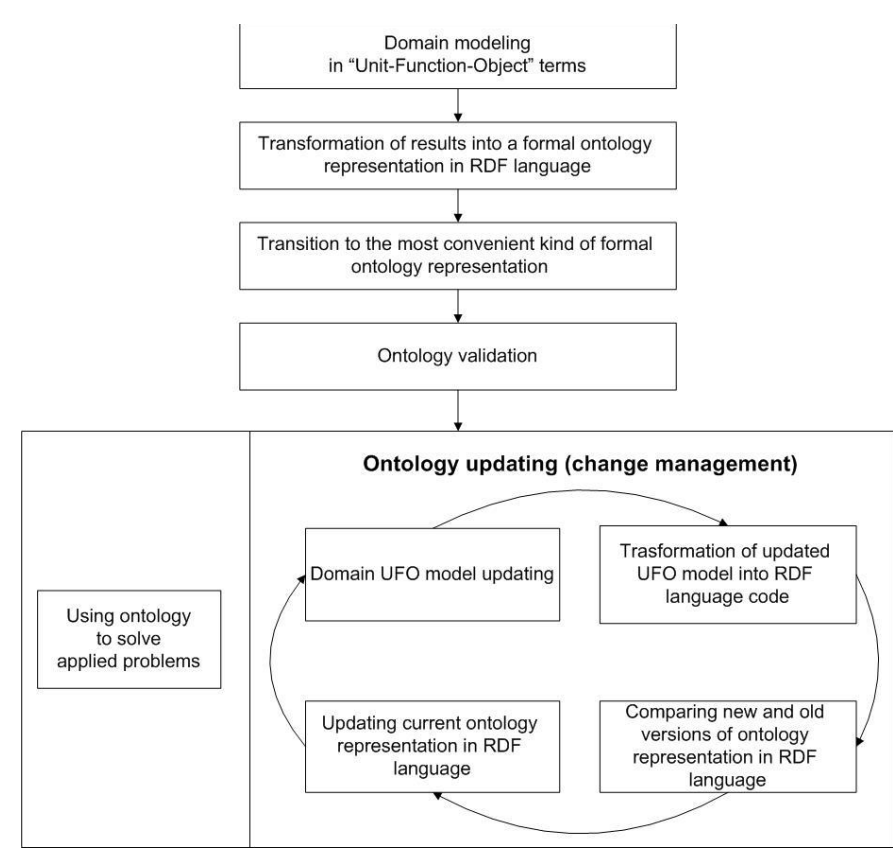

Fig. 1. The diagram of the ontology building method based on UFO analysis results.

This method involves using a model developed like result of UFO analysis as a source of information (facts) for including in the future ontology. This approach allows us to use the extracted earlier and, accumulating experts knowledge results of the domain modeling and analysis without the need for laborious and lengthy procedures for selecting concepts and establishing links between them. The UFO model, which is taken as a basis, can be updated and supplemented in the future, which makes it possible to ensure the relevance of the data source for building an ontology.

The next component of the method of ontology building based on integration with the UFO approach is the extraction of information (facts) about the domain from the original system-object model. The UFO model is investigated for the certain facts provided by the general classification [12]. The revealed facts are used as axioms, which form the basis of the future ontology.

The transformation stage is next, when the extracted from the UFO domain model facts are transformed from the original representation into the construction of specialized languages intended for the ontologies formal description (RDF, OWL). Because the classification of extracted facts strictly determines the initial data and the transformation in the construction of ontology representation languages takes into account the syntax of the chosen language (for example, RDF), the 
transformation process can be represented as an algorithm and subsequently automated.

The ontology representation language constructions created during the transformation are written to the resulting file, which serves as a formal representation of the ontology created on the basis of the UFO domain model. The received ontology view is subjected to the evaluation and validation procedure, with the verification for the absence of syntactic and semantic errors and consistency of data from the point of view of the UFO approach.

After obtaining a formal representation of the ontology, formed on the basis of the results of the UFO analysis of domain, the transition to the most optimal (in the context of the problem being solved) method of representing ontologies is presented: as a graphs, as a code in one of the notations of the RDF, OWL or other syntax. The resulting ontology is used to solve applied problems; its actualization and development is carried out in parallel.

Therefore, in comparison with the well-known approaches and methods of ontologies building, the proposed method has several advantages:

- correspondence to the current conventional life cycle of an ontology;

- formalized technique for selecting concepts that are included in the ontology;

- application independence;

- problem of an optimal criterion of a break in ontology development and actualization cycle is not actual;

- predictability of the time required to create an ontology;

- compliance with the principle of extensibility due to the possibility of updating the ontology while the original UFO model is updated;

- relatively small number of iterations of the ontology building process.

\section{Particularity of validation of ontologies built on UFO domain models}

In the process of ontology building using the proposed method, as well as preserving its completeness and relevance by change management it is necessary to validate the ontology. Validation in this case means an assessment of the consistency of the ontology and correspondence its formal representation with the regulated rules of sufficient language (format).

In literature [13] we can find descriptions for the three main approaches to the ontology evaluation and validation, based on the evaluation of the ontology evolution during the time, on rules and on metrics. We will use the approach based on rules.

So, the correct ontology built on the basis of the UFO domain model must match with a some rules. The Semantic Web Rule Language (SWRL) is used to formally record these rules. SWRL is a specialized language that allows to develop and include output rules directly in the ontology code. SWRL has one of notations named Human Readable Syntax, which is easy for human perception.

The rules written by the use of SWRL Human Readable Syntax, consist of an antetsendent and a consequent. A consequent is true when an antetsendent is empty or all of its components are true. In consequent and in an antetsendent we can use properties (predicates), which are included into ontology, and variables (they are written with a question mark).

The follow list is a specific rules in the Human Readable Syntax notation of the SWRL language for validating an ontology based on the UFO model. In these rules we use symbols for variables: ? $L$ for some link in UFO model, ? $L C 1$ and ? $L C 2$ for different classes from classification of links for analyzed UFO model, ?x, ?y for some concepts in ontology, ?id for concept identifier like its property, ?f , ?fl, ?f2 for Function like a part of some UFO element, ?u1 and ?u2 for Units like a parts of UFO elements, ?o, ?o1, ?o2 for Objects like a parts of UFO elements, ? $N$ for 'name' property value.

- Every link must have class name(1): hasClass(?L, null) $\Rightarrow$ hasNoLinkClass(?L);

- Every link must have only one class name (2): hasClass(?L, ?LC1) ^hasClass(?L, ?LC2) $\Rightarrow$ hasManyLinkClasses(?L);

- Ontology must not include several entities with the same value of identifier (ID) (3):

$$
\text { rdfs : hasID(?x, ?id) } \wedge \text { rdfs : hasID(?y, ?id) } \Rightarrow
$$

hasSameID $(? x, ? y)$

- One Function cannot balance more then one Unit (4):

balances(?f, ?u1) ^balances(?f, ?u2) $\Rightarrow$ balancesManyNodes(?f);

- One Object cannot represent more then one Unit (5):

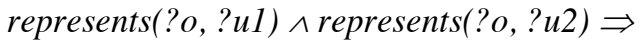
representsManyNodes(?o);

- В онтологии не должно быть нескольких объектов с одинаковым именем(значением свойства Name) (6):

Name(?o1, ?N) ^Name(?o2, ?N) $\Rightarrow$ sameNameObjects(?o1, ?o2);

- Ontology must not include several Functions with the same name (with the same value of property 'Name') (7):

Name(?f1, ?N) ^ Name(?f2, ?N) $\Rightarrow$ sameNameFunctions(?f1, ?f2);

- Ontology must not include several Units with the same notation (with the same value of property 'Notation') (8): 
Notation(?u1, ?N) $\wedge \quad$ Notation(?u2, ?N) $\Rightarrow$ sameNotationNodes(?u1, ?u2).

The validation with the use of these specified rules of the developed on the basis of the UFO model ontology allows reducing the number of logical errors in the resulting ontology.

\section{UFO approach and ontologies mapping}

Taking into account the aforesaid, it can be concluded that integration of the UFO approach means and ontology engineering allows to solve some actual problems in the sphere of applied ontologies construction and actualization. However, up to the present moment, the prospects of using the system-object approach to solve the problem of mapping and merging two available ontologies have not been considered in the literature. Nevertheless, the successful experience of using the system-object approach tools in the process of building new ontologies allows us to put forward a hypothesis about the possibility of using such tools for ontology mapping too.

Let's describe the main components of the proposed method of mapping and merging ontologies using the principles of the UFO approach:

1) Transformation of the basic (first) ontology into a formal representation of the UFO model.

2) Transformation of the updated (second) ontology to the formal representation of the UFO model.

3) On-object compare of formal representations of received UFO models and establishment of differences / correspondences, specifically:

- UFO elements and its attributes;

- Functions of UFO elements;

- using links classification;

- existing in model links;

- Unit ports like one of the UFO elements parts.

4) Formulation and transformation in the construction of ontology formal description languages facts, that characterize the found differences between UFO models.

5) Inclusion in the resulting ontology all structures that correspond to the facts present in both UFO models, i.e. the same fragments of compared ontologies.

6) Inclusion in the resulting ontology all structures that correspond to the facts missing in one, but existing in another of compared ontologies.

In fact, comparing the number of UFO elements and analyzing the differences in the values of their attributes in the obtained models allows us to identify differences in the sets of concepts and the relationships between them in comparable ontologies. Identifying the differences between UFO models and formulating the facts that characterize these differences (with according to the proposed in [12] classification of the facts for building ontologies), we get a difference between analyzed ontologies, which can be useful for analyzing changes that occurred, for example, in the process of actualization of ontology.

To solve the problem of merging ontologies, the resulting description in the formal language includes constructions corresponding to the facts-differences between the initial ontologies. In this case, both analyzed ontologies are considered equivalent and are used to create a new, more complete ontology.

Thus, the means of the UFO approach can be applied not only for directly building an individual applied ontology, but also for mapping and merging existing ontologies. At the moment, the authors are working towards a more detailed research of the applicability of the UFO approach for the purpose of mapping ontologies and developing specific techniques and algorithms for comparing two ontologies with an intermediate transformation into formal descriptions of UFO models of domains.

\section{REFERENCES}

[1] T.R. Gruber, "A translation approach to portable ontology specification", Knowledge Acquisition, vol.5(2), 1993, pp.199-220.

[2] S.V. Kozlov, A.F. Tuzovsky, S.V. Chirikov, V.Z. Yapmolsky, "Using ontologies in organization knowledge management systems", Bulletin of the Tomsk Polytechnic University, vol.3, 2006, pp.180-184.

[3] M. Gruninger, J. Lee, "Ontology Applications and Design. Introductory article to a special issue on Ontology Engineering", Communications of the ACM, vol. 45(2), 2002, pp.39-41.

[4] T.A. Gavrilova, V.F. Khoroshevsky, " Knowledge bases of intellectual systems. Textbook for high schools", Piter, 2000.

[5] A.P. Karpenko, R.S. Sukhar, "Methods of ontotlogies mapping. Overview", Engineering education, vol.01, January 2009.

[6] S.I. Matorin, "About the new method of the systemological analysis coordinated with the procedure of object-oriented design. Part 1", Cybernetics and systems analysis, vol.4, 2001, pp.119-132.

[7] S.I. Matorin, "About the new method of the systemological analysis coordinated with the procedure of object-oriented design. Part 2", Cybernetics and systems analysis, vol.1, 2002, pp.118-130.

[8] S.I. Matorin, O.A. Zimovets, "Formalized visual modeling of administrative procedures", Applied informatics, vol.2, 2012, pp.100110

[9] S.I. Matorin, A.S. Popov, V.S. Matorin, "Modeling of organizational systems with the new "Unit-Function-Object" approach view", NTI, 2, vol.1, 2005, pp.1-8.

[10] S. Cranefield, M. Purvis, "UML as an ontology modelling language", Proceedings of the Workshop on Intelligent Information Integration, 16th International Joint Conference on Artificial Intelligence (IJCAI-99) Germany, University of Karlsruhe, 1999, pp.46-53.

[11] A.A. Kondratenko, S.I. Matorin, "Ontologies building on the basis of Unit-Function-Object models", Artificial Intelligence and Decision Making, vol.1, 2016, pp. 47-56.

[12] A.A. Slobodyuk, "An extended classification of the facts extracted from the UFO model for the purpose of a domain ontology building", Proceedings of the Infocom-6, part II, North-Caucasian federal university, April 2014, pp. 404-406.

[13] S. Tartir, I. Arpinar, A. Sheth, "Ontological Evaluation and Validation", Theory and Applications of Ontology (TAO), vol.2, 2008. 